\title{
Cyclic Arrayed Waveguide Grating Devices With Flat-Top Passband and Uniform Spectral Response
}

\author{
Hung-Chih Lu and Way-Seen Wang, Member, IEEE
}

\begin{abstract}
Cyclic arrayed waveguide grating (AWG) devices with flat-top passband and uniform spectral response are proposed. Each access waveguide of the proposed cyclic AWG consists of a multimode interference region and a taper waveguide. Simulation results show that the 1-dB bandwidth of the proposed device is larger than 0.44 times the channel spacing and the corresponding nonuniformity is smaller than $1 \mathrm{~dB}$.
\end{abstract}

Index Terms-Arrayed waveguide grating (AWG), cyclic, flattop, spectral response.

\section{INTRODUCTION}

$\mathbf{O}$ PTICAL networks are widely used in modern communication systems. The use of optical devices with good characteristics such as wide bandwidth, compact size, high fabrication tolerance, stable output, polarization independence, low power loss, etc., is becoming more and more important. Arrayed waveguide gratings (AWGs) are the key devices for wavelengthdivision multiplexers/demultiplexers and wavelength routers [1] in backbone optical networks. In recent years, fiber-to-the-home has been built gradually, the use of cyclic AWG in any wavelength bands is paid attention to and studied for its expansibility.

However, the passband is not flat-topped and the spectral response is not uniform for conventional cyclic AWG. Those require a light source of high accurate wavelength in order to have several signals with different wavelengths be multiplexed by an AWG and coupled into the same access fiber. In principle, the spectral response of cyclic AWG has a 3-dB nonuniformity [1]. Thus, variable optical attenuators are necessary to equalize the powers between the output signals [2], [3]. It would then be too expensive to use a variable optical attenuator for each channel in an AWG of 200 channels for commercial application.

Therefore, flat-topped passband [4] and uniformed spectral response are of great importance in order to have signals be equally multiplexed/demultiplexed by the same AWG and coupled into an access fiber.

To reduce the transmission loss, multimode interference (MMI) structures with taper access waveguides were reported in splitter and coupler applications [5], [6]. In this paper, the MMI structure with a taper access waveguide is used between the second slab and the output access waveguides of a cyclic

Manuscript received May 26, 2007; revised September 6, 2007.

The authors are with the Department of Electrical Engineering and the Graduate Institute of Photonics and Optoelectronics, National Taiwan University, Taipei 106, Taiwan, R.O.C. (e-mail: wswang@cc.ee.ntu.edu.tw).

Digital Object Identifier 10.1109/LPT.2007.910090

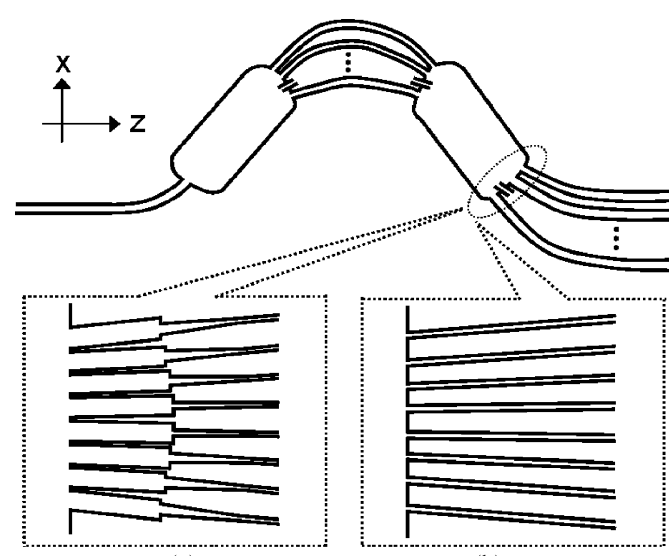

(a)

(b)

Fig. 1. Cyclic AWG (a) proposed and (b) conventional.

AWG to improve the device characteristics. With a proper design of each access waveguide, the calculated spectral response is found uniformed and the passbands are flat-topped.

\section{PROPOSED CYCLIC AWG}

The proposed cyclic AWG, shown in Fig. 1(a), consists of a cyclic AWG and many MMI structures with taper access waveguides. The MMI structures are used to have the passbands flattopped, whereas the linear-taper waveguides are introduced to obtain a uniformed spectral response.

\section{A. Cyclic AWG}

The cyclic AWG structure consists of an input waveguide, a first slab waveguide, arrayed waveguides, a second slab waveguide, and output access waveguides as shown in Fig. 1(b). The path length difference $\Delta L$ between adjacent arrayed waveguides can be written as [1]

$$
\Delta L=\frac{c}{\widetilde{N}_{g} \cdot N \cdot \Delta f}
$$

where $N$ is the number of channels, $\Delta f$ is the channel spacing, and $\widetilde{N}_{g}$, the group index of waveguide mode, is equal to

$$
\widetilde{N}_{g}=N_{g}+f \cdot \frac{d N_{g}}{d f}
$$

where $N_{g}$ is the effective index of waveguide mode. The radius of outer Rowland circle $R_{a}$ is

$$
R_{a}=\frac{d_{a}}{\Delta \alpha}
$$




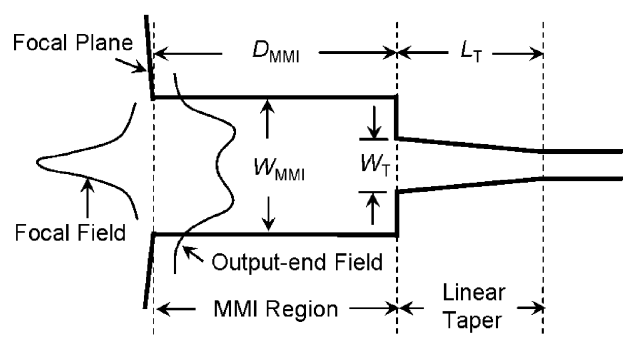

(a)

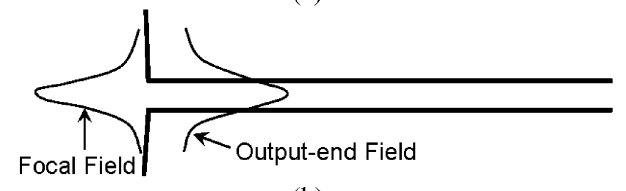

(b)

Fig. 2. Output-end structure of cyclic AWG. (a) MMI structure with a taper (proposed) and (b) conventional.

where $d_{a}$ is the lateral spacing (center-to-center) of arrayed waveguides and $\Delta \alpha$, the divergence angle, is given by

$$
\Delta \alpha=\frac{\tilde{N}_{g} \cdot \Delta L}{f_{c} \cdot N_{\mathrm{FPR}} \cdot D}
$$

where $f_{c}$ is the central frequency, $N_{\text {FPR }}$ is the slab mode index in the free propagation region, and $D$ is the dispersion of array and can be written as

$$
D \equiv \frac{d s}{d f}=\frac{d s}{d \lambda} \cdot \frac{\lambda^{2}}{c}
$$

where $d s$ is the lateral displacement of focal spot (center-tocenter).

According to the operational principle of AWG, signals of different wavelengths are focused on the same focal plane of the second slab waveguide. In general, the field distribution of the output access waveguide of the AWG is a Gaussian-like function as shown in Fig. 2(b). Thus, signals focused at the center of the output access waveguide give rise to the largest transmission, and elsewhere, smaller transmission. Therefore, the passband of the spectra is Gaussian-like.

\section{B. MMI Structure With Taper Access Waveguide}

In order to compensate both the Gaussian-like distribution of the passband and the nonuniformity of the output spectrum, an MMI coupler with taper access waveguide is proposed. The proposed MMI coupler is essentially a truncated $1 \times 2 \mathrm{MMI}$ power splitter that does not have two distinct peak powers. The coupling length of a $1 \times 2 \mathrm{MMI}$ splitter $L_{\mathrm{MMI}}$ can be written as [7]

$$
L_{\mathrm{MMI}} \cong \frac{n_{g}}{2 \lambda} \cdot W_{\mathrm{MMI}, e}^{2}
$$

where $n_{g}$ is the waveguide index, $\lambda$ is the signal wavelength, and $W_{\mathrm{MMI}}$ and $W_{\mathrm{MMI}, e}$ are the physical and the effective width of interference region. The interference length of the MMI region $D_{\mathrm{MMI}}\left(D_{\mathrm{MMI}}<L_{\mathrm{MMI}}\right)$ can be shortened to obtain two overlapped rather than two distinct peak power distributions as shown in Fig. 2. When the incident Gaussian beam passes through such a truncated $1 \times 2$ MMI splitter, the output power

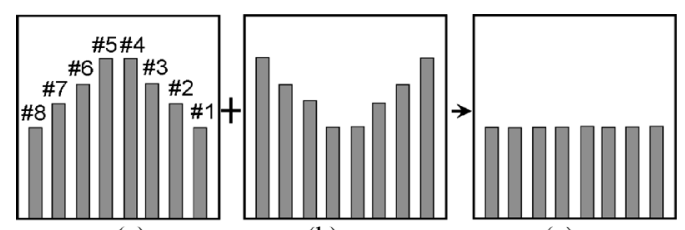

(a)

(b)

(c)

Fig. 3. Illustration of relative transmission of each channel by selecting a suitable value of $W_{T}$. (a) With MMI structure only; (b) with taper only; (c) with both MMI structure and a taper (compensated results).

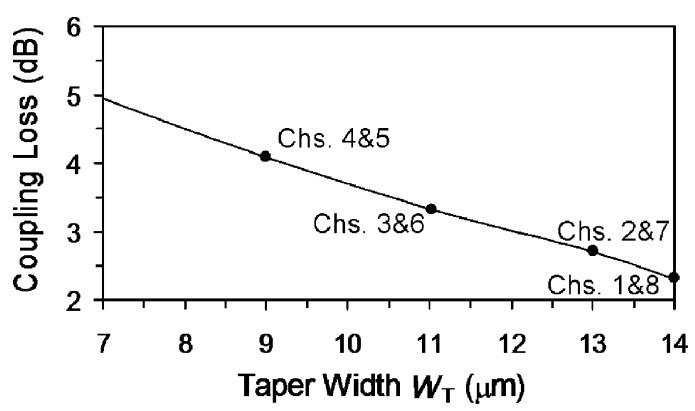

Fig. 4. Calculated coupling loss of each tapered output channel.

distribution of each passband can be significantly flattened due to opposite power distributions. Therefore, the output power distributions of passbands can be flat-topped though the entire spectrum is not uniform, as shown in Fig. 3(a).

To have the spectrum uniformed, the width of each taper access waveguide $W_{T}$ can be properly chosen for a specific transmission, as shown in Fig. 3(b). Thus, the entire spectrum can be uniformed, as shown in Fig. 3(c).

\section{Simulation Results}

As silicon photonics are paid much attention [8], the waveguide structure of silicon-on-insulator (SOI) is chosen for study. The indexes of silicon waveguide and silica cover are chosen as 3.4 and 1.45. For practical application, a $1 \times 8200-\mathrm{GHz}-$ spaced cyclic AWG is proposed and simulated by the finitedifference beam propagation method. Assume the waveguide width is $5 \mu \mathrm{m}$ and the etch depth and slab thickness are both equal to $3 \mu \mathrm{m}$. The central wavelength is set to be $1.55 \mu \mathrm{m}$. The corresponding free-spectral range (FSR), defined as $N \Delta \lambda$, is found to be $12.8 \mathrm{~nm}$. The grating order $\lambda / \mathrm{FSR}$ is equal to 121 . The values of $d s$ and $d_{a}$ are set to be 26 and $18.5 \mu \mathrm{m}$, respectively. From (1), $\Delta L$ can be calculated to be $55.23 \mu \mathrm{m}$. From (3), $R_{a}$ is found to be $8.44 \mathrm{~mm}$ and is chosen as the slab focal length.

The two-peak field distribution of the proposed cyclic AWG with MMI structure and taper access waveguide for flap-top compensation can be chosen with a specific value of $D_{\mathrm{MMI}}$. Moreover, the transmission loss of the proposed cyclic AWG with MMI structure and taper access waveguide is shown in Fig. 4. As can be seen, the transmission loss is linearly decreased with an increasing $W_{T}$. A proper transmission loss for each channel of AWG can be realized by choosing a suitable $W_{T}$. As the transmission loss of each channel can be individually selected to make the spectral response uniform on the same chip, 


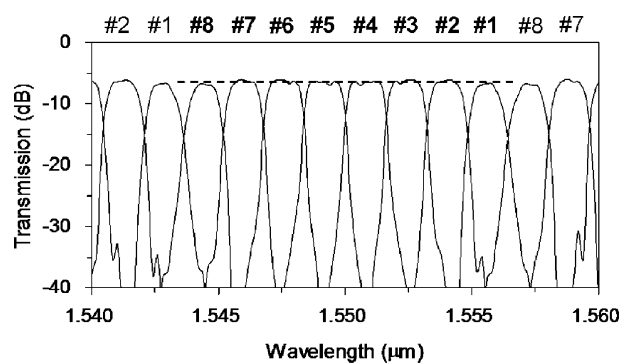

(a)

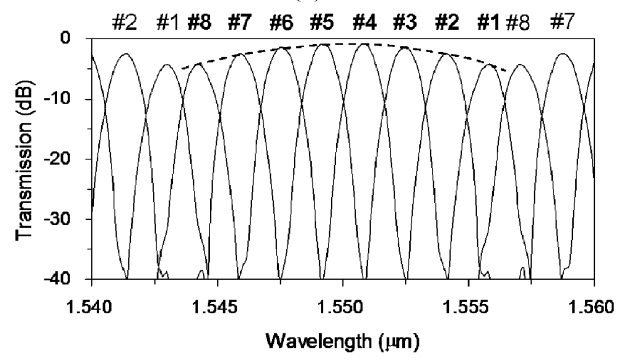

(b)

Fig. 5. Spectra of cyclic AWG (a) proposed and (b) conventional.

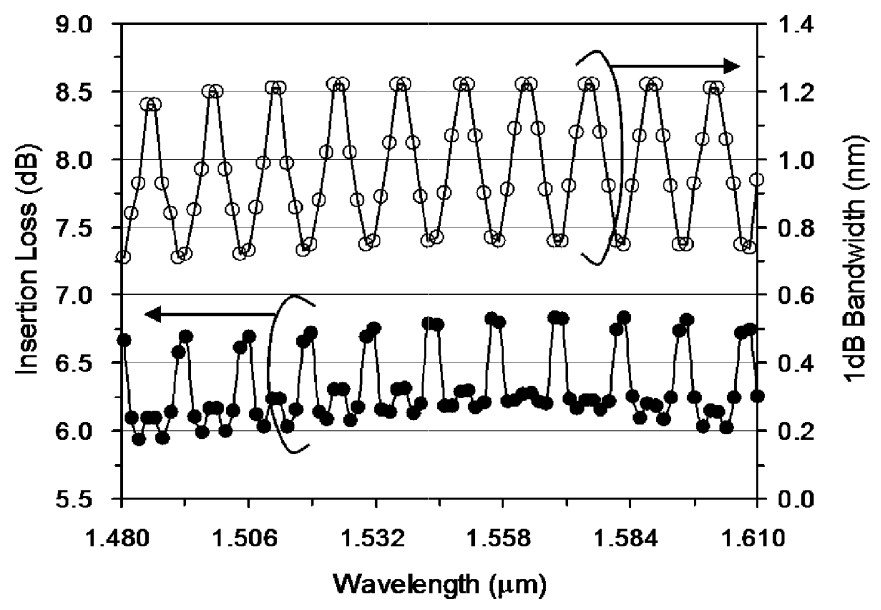

Fig. 6. Variation of insertion loss and 1-dB bandwidth of proposed cyclic AWG for over-all operating wavelength range.

several devices can be integrated to become a module-on-a-chip such as wavelength cross connect and wavelength-division-multiplexing modulator, etc. The proper values of $D_{\mathrm{MMI}}$ are equal to $635,630,620$, and $570 \mu \mathrm{m}$ and the corresponding values of $W_{T}$ are equal to $9,11,13$, and $14 \mu \mathrm{m}$, respectively. Note that the values of $W_{\mathrm{MMI}}$ and $L_{T}$ are fixed to be 24 and $500 \mu \mathrm{m}$, respectively for simplicity.

The spectra of the proposed $1 \times 8200-\mathrm{GHz}$-spaced cyclic AWG are shown in Figs. 5(a) and 6. As can be seen from figures, the 1-dB bandwidth are all larger than 0.44 times the channel spacing and the nonuniformity is smaller than $1 \mathrm{~dB}$ for the operational wavelength range is varying from 1.48 to $1.61 \mu \mathrm{m}$, which is wide enough for ten cyclic bands. For comparison, the spectra of the conventional cyclic AWG are shown in Fig. 5(b). Obviously, the spectral response of the proposed cyclic AWG is uniformed and all the passbands are nearly flat-topped as compared to those of the conventional cyclic AWG.

Nevertheless, the total transmission loss of the proposed cyclic AWG with MMI structures and taper access waveguides is increased. For example, the increased loss is about $2.3 \mathrm{~dB}$ when the taper width is $14 \mu \mathrm{m}$, but becomes $4.1 \mathrm{~dB}$ when the taper width is $9 \mu \mathrm{m}$, as shown in Fig. 4 . That is an inevitable trade-off for the compensation of the nonuniformity of cyclic AWG. However, it can be easily overcome by an erbium-doped fiber amplifier.

Obviously, the bandwidths of passbands are not the same. However, the 1-dB bandwidths of the passbands are all larger than 0.44 times the channel spacing, which are better than the performance criterion of GR-1209-CORE (0.35 times the channel spacing) [9].That is more important for practical application.

\section{CONCLUSION}

Cyclic AWG devices with flat-top passband and uniform spectral response can be obtained by using an MMI region and a taper access waveguide for each output access waveguide of the cyclic AWG. Simulation results show that the 1-dB bandwidth can be as wide as 0.44 times the channel spacing and the nonuniformity is smaller than $1 \mathrm{~dB}$. Details of the application will be of great interest for future study.

\section{REFERENCES}

[1] M. K. Smit and C. V. Dam, "Phasar-based WDM-devices: Principles, design, and applications," IEEE J. Sel. Topics Quantum Electron., vol. 2, no. 2, pp. 236-250, Jun. 1996.

[2] H. Uetsuka, "AWG technologies for dense WDM applications," IEEE J. Sel. Topics Quantum Electron., vol. 10, no. 2, pp. 393-402, Apr. 2004.

[3] C. R. Doerr, H. K. Kim, L. W. Stulz, M. Cappuzzo, L. Gomez, A. Paunescu, E. Laskowski, L. Boivin, and S. Shunk, "40-channel multi/demultiplexer with dynamic passband shape compensation," IEEE Photon. Technol. Lett., vol. 13, no. 7, pp. 690-692, Jul. 2001.

[4] M. R. Amersfoort, J. B. D. Soole, H. P. LeBlanc, N. C. Andreadakis, A. Rajhel, and C. Caneau, "Passband broadening of integrated arrayed waveguide filters using multimode interference couplers," Electron. Lett., vol. 32, no. 5, pp. 449-451, Feb. 1996.

[5] Q. Wang, S. He, and L. Wang, "A low-loss Y-branch with a multimode waveguide transition section," IEEE Photon. Technol. Lett., vol. 14, no. 8, pp. 1124-1126, Aug. 2002.

[6] M. T. Hill, X. J. M. Leijtens, G. D. Khon, and M. K. Smit, "Optimizing imbalance and loss in $2 \times 23$-dB multimode interference couplers via access waveguide width," J. Lightw. Technol., vol. 21, no. 10, pp. 2305-2313, Oct. 2003.

[7] L. Soldano and E. Pennings, "Optical multi-mode interference devices based on self-imaging: Principles and applications," J. Lightw. Technol., vol. 13, no. 4, pp. 615-627, Apr. 1995.

[8] T. Bestwick, "ASOC-A silicon-based integrated optical manufacturing technology," in Electron. Component Technol. Conf., May 1998, pp. 566-571.

[9] GR-1209-CORE, no. 2, Feb. 1998. 